\begin{tabular}{|c|c|}
\hline Title & A nalysis of time course drug response in rat cardiomy ocytes cultured on a pattern of islands \\
\hline Author(s) & Okumura, Sho; Hirano, Y u; Maki, Y oshiy uki; Komatsu, Y asuo \\
\hline Citation & $\begin{array}{l}\text { A nalyst, 143(17), 4083-4089 } \\
\text { https://doi.org/10.1039/c8an01033a }\end{array}$ \\
\hline Issue Date & 2018-09-07 \\
\hline Doc URL & http:/hdl.handle.net/2115/75436 \\
\hline Type & article (author version) \\
\hline Additional Information & There are other files related to this item in HUSCAP. Check the above URL. \\
\hline File Information & 2018_A nalyst_Okumura.pdf \\
\hline
\end{tabular}

Instructions for use 


\title{
Analysis of time-course drug response in rat cardiomyocytes cultured on a pattern of islands
}

\author{
Sho Okumura, ${ }^{1,2}$ Yu Hirano, ${ }^{2,3}$ Yoshiyuki Maki, ${ }^{1}$ Yasuo Komatsu ${ }^{2,3,}$ * \\ 1 Cosmo Bio Co., Ltd., 2 Grad. Sch. of Life Sci., Hokkaido Univ.,3 AIST
}

\begin{abstract}
1 Cosmo Bio Co., Ltd., Hokkaido, 047-0261, Japan
2 Graduate School of Life Science, Hokkaido University, 060-0810, Sapporo, Japan

3 Bioproduction Research Institute, National Institute of Advanced Industrial Science and Technology (AIST), Hokkaido, 062-8517, Japan.

*To whom correspondence should be addressed: E-mail: komatsu-yasuo@aist.go.jp; Tel: +81-11-857-8437, Fax: +81-11-857- 8954
\end{abstract}




\begin{abstract}
We previously reported the kinetic analysis of cardiomyocyte beating using scanning electrochemical microscopy (SECM). In this study, a stage-top incubator and a capillary micropipette (MP) for delivering drugs were assembled with an SECM, and the responses of rat cardiomyocytes were analyzed under cultural environment after drug stimulation. When adenosine triphosphate (ATP) was delivered to synchronously beating cardiomyocytes, beating acceleration effect of ATP was counteracted by synchronously beating network in the culture dish. In contrast, cardiomyocytes cultured on a pattern of island in a culture dish showed fluctuations in the duration of beating upon addition of ATP. We also examined the effect of the cardiotoxic agent astemizole on cardiomyocytes and successfully detected motion fluctuations. Therefore, drug stimulation via MP and beating measurement by SECM are effective for the evaluation of drug candidates through analysis of time-course beating motion fluctuations of the cardiomyocytes.
\end{abstract}




\section{Introduction}

Many drugs have been withdrawn from commercial markets because of potential risks to patients. ${ }^{1,}$

2 Most of the risks are cardiotoxic without regard to specific diseases. ${ }^{1,3}$ Therefore, in the developmental phase of drug development, analyses of cardiomyocyte responses to candidate agents are important to evaluate the drug's pharmacological and toxicological properties. ${ }^{4}$ In many cases, the measurement of action potentials by a microelectrode array has been used to analyze cardiotoxicity. ${ }^{5}$ However, electrophysiological methods cannot eliminate non-beating cells and non-cardiomyocytes, which may cause errors in the data. By using a fluorescent calcium indicator, the imaging of calcium signaling is also used to examine cardiomyocyte contraction. ${ }^{6,7}$ However, fluorescent indicators may cause photodamage. ${ }^{8}$ These studies were focused on the physiological processes of cardiomyocytes and did not reveal information on the beating properties of cardiomyocytes.

Atomic force microscopy (AFM) is used to analyze the mechanical properties of cardiomyocytes. ${ }^{9}$ AFM is not invasive, but a small cantilever probe makes direct contact with the cardiomyocytes. An impedance-based assay has also been used to analyze the beating properties of cardiomyocytes. ${ }^{10}$ The impedance signal varies according to the movement of cardiomyocytes that are seeded on an electrode. These signals are a mixture of beating and non-beating cells. Recently, video-based analytical methods have been applied to evaluate the beating kinetics of cardiomyocytes. ${ }^{11-14}$ The non-invasive movies are analyzed by several different computational methods. We have also reported a scanning electrochemical microscopy (SECM)-based system that could analyze the contraction kinetics and oxygen consumption of cultured cardiomyocytes. ${ }^{15}$ SECM, which is a kind of scanning probe microscopy technique, is used for the analysis of various biomaterials such as enzyme activity, ${ }^{16,17}$ cellular metabolism, ${ }^{18-20}$ and cellular status. ${ }^{21,22}$ Because SECM is a non-invasive and high-resolution method using a microelectrode, it can measure both the shape and motion of a single living cell ${ }^{23-26}$ and is therefore well suited for the analysis of cardiac beatings.

The activity of cardiomyocytes is significantly sensitive to temperature or $\mathrm{pH}$ changes. ${ }^{27,28}$ Thus a stage-top incubator that consists of a sealed chamber is essential for the accurate analysis of

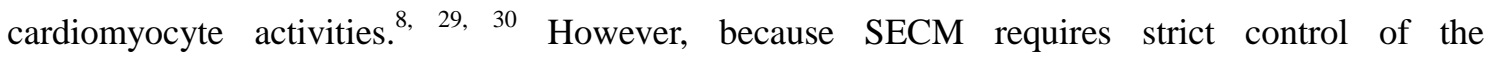
microelectrode position at a resolution in the order of nanometer, it is difficult to perform SECM measurements in a sealed chamber. Therefore, at present only a temperature controlled-plate has been used for SECM experiments on living cells. ${ }^{15,19,23}$

Because the drug response of cardiomyocytes appears immediately after drug stimulation, ${ }^{31}$ motion measurement and drug stimulation should be performed simultaneously in the analysis of initial drug responses. However, it is difficult to perform SECM measurements during drug stimulation because the SECM measurement is easily perturbed by conventional drug stimulation methods that add the 
drug solution to the entire culture medium. We previously reported a SECM-based micropipette (MP) system for localized drug delivery that targets a single cell. ${ }^{32}$ In this system, the SECM measurement was performed with drug delivery and the microelectrode current signals were used to control the amount of drug administered. It is expected that this system might be applicable to the analysis of cardiac beating.

This study aims to develop a new system for the evaluation of the drug response of cardiomyocytes. We constructed a sealed chamber on an SECM to maintain a stable culture environment. We also fit a local drug delivery system to the chamber. We confirmed that the SECM-MP system could stimulate cardiomyocytes, then analyzed beating fluctuations using ATP solution. Furthermore, we evaluated the effect of astemizole, a cardiotoxic agent, by analyzing the cell contraction and relaxation behaviors. 


\section{Experimental}

\section{Chemicals}

Adenosine triphosphate (ATP) and astemizole were purchased from Sigma-Aldrich (USA). ATP was dissolved in phosphate buffered saline (PBS(-)). Astemizole was dissolved in dimethyl sulfoxide (DMSO) to procedure a stock solution, and diluted with PBS(-) before loading into the MP system.

\section{Cell culture}

All animal procedures were performed in accordance with the "Fundamental Guidelines for Proper Conduct of Animal Experiment and Related Activities in Academic Research Institutions” stipulated by the Ministry of Education, Culture, Sports, Science and Technology and approved by the animal experiment committee of Cosmo Bio Co., Ltd. Primary rat cardiomyocytes were cultured as previously described. ${ }^{15}$ Briefly, cardiomyocytes prepared from neonatal rat ventricles (Cosmo Bio, Japan) were seeded on $35-\mathrm{mm}$ dishes that were coated with collagen at a concentration of $2 \times 10^{5}$ cells/dish. These cells were then incubated in cardiomyocyte culture medium (Cosmo Bio) at $37^{\circ} \mathrm{C}$ in a humidified atmosphere containing $5 \% \mathrm{CO}_{2}$. The medium was changed two days after seeding. For SECM experiments, we used spontaneously beating cells within 25 days in a culture. Before making a beating measurement, the culture medium was replaced with $2 \mathrm{ml} / \mathrm{dish}$ of serum-free medium that contained $1 \mathrm{mM}$ potassium ferrocyanide $\left(\mathrm{K}_{4}\left[\mathrm{Fe}(\mathrm{CN})_{6}\right]\right)$ that is an electron mediator of SECM measurement and $2 \%$ Knockout ${ }^{\mathrm{TM}}$ serum replacement (Thermo Fisher Scientific, USA) in DMEM/F-12 medium (Thermo Fisher Scientific, USA).

\section{Cell patterning}

CYTOOchips ${ }^{\mathrm{TM}}$ Arena FN (CYTOO, USA) was placed on the bottom of a 35-mm dish and fixed at four corners of the glass chip using vacuum grease. Then cardiomyocytes were seeded in the same way as normal culture methods. For drug delivery experiments, we used relatively short-term cultured cardiomyocytes because the cell pattern was lost in long-term cultures. Serum-free medium, as described above, was used for the beating measurement.

\section{SECM instrumentation}

The basic part of the SECM instrument was built in our laboratory according to our previous studies $^{15}$ with some of modifications. In brief, the central part of our SECM setup comprised an inverted phase-contrast microscope (NIKON, Japan), a piezomotor positioning system (Nano control, Japan), and a potentiostat (Hokuto Denko, Japan). The XYZ-axis stepping-motors (Suruga Seiki, Japan) were introduced for electrode positioning. The software of the SECM system was constructed using LabView (National Instrument, USA). We used a Pt microdisk electrode (5- $\mu$ m radius) as the 
electrochemical probe for SECM measurements and an Ag/AgCl electrode (Hokuto Denko, Japan) as the reference and auxiliary electrode.

The MP system was set up on the SECM system as previously described. ${ }^{32}$ Briefly, the microinjector (Narishige, Japan) pressure was controlled by a stepping-motor. The MP position was also controlled by XYZ-axis stepping-motors. The MPs were fabricated from borosilicate capillaries (o.d./i.d.=1.0/0.6 mm with an inner filament) using a micropipette puller.

\section{Environment maintenance system}

A temperature controlled-plate (Tokai Hit, Japan) was set on the piezomotor system. The piezomotor system was surrounded by a metal base. The chamber was made from polyvinyl chloride (PVC) with dimensions of $200 \mathrm{~mm} \times 260 \mathrm{~mm} \times 90 \mathrm{~mm}$ (length $\times$ width $\times$ height). The chamber had a small window sealed by a rubber film for the microelectrode shaft. A small box, that also had a sealed small window, was set on top of the PVC chamber for the MP. The chamber was put on the metal base and the bottom of the chamber was connected to the temperature controlled-plate via a rubber film (Fig. 1A). A $\mathrm{CO}_{2}$ sensor (Kenis, Japan), and a temperature and humidity sensor (T\&D Corporation, Japan) were placed in the PVC chamber. $\mathrm{CO}_{2}$ gas was mixed with air to $5 \%$ using a gas mixer (Okolab, Italy) and humidified through a bubbling humidifier (Tokken, Japan), then drawn into the chamber. The PVC chamber was also connected to a HEPA filter unit to clean the inside then the door was opened.

\section{Measurement of cardiomyocyte beating by SECM}

The measurement procedures of beating cardiomyocytes by SECM were previously described. ${ }^{15}$ Briefly, we applied a potential of $0.5 \mathrm{~V}$ to the probe electrode for ferrocyanide oxidation. We positioned the microelectrode tip $9 \mu \mathrm{m}$ away from a cell using an approach curve measurement, and subsequently measured the oxidation current of the ferrocyanide with a time resolution of $10 \mathrm{~ms}$. To analyze the beating of the cardiomyocytes, a plot of the current response was studied using software that was developed in LabView. In addition to a previous version of the software that could calculate a peak-to-peak interval time, a beating rate and an average waveform, a new program in this study could also extract the current data within an optional time scale and correct for changes in background current due to the reduced sensitivity of microelectrode induced by metabolites within the cells. The time of contraction and relaxation were calculated as previously described. ${ }^{15}$

\section{Control of drug delivery with $M P$}

The MP positioning and delivery were controlled as previously described. ${ }^{32}$ Briefly, the MP delivered a drug solution which did not contain any electron mediators upstream from the microelectrode into a medium containing $1 \mathrm{mM} \mathrm{K}_{4}\left[\mathrm{Fe}(\mathrm{CN})_{6}\right]$. The $\mathrm{K}_{4}\left[\mathrm{Fe}(\mathrm{CN})_{6}\right]$ oxidation currents 
decreased depending on the delivery rate. The MP delivery was controlled with a feedback control system between the current signals and the microinjector pressure. The MP was positioned diagonally above the microelectrode to target cells (Fig. 1A). Before and after delivery, the MP tip was kept away from a target cell to prevent unexpected delivery caused by leakage from the MP tip. These operations were controlled using software developed with LabView. The program for controlling the MP system and the program for measuring the cardiomyocyte beating were operated cooperatively. 


\section{Results and Discussion}

\section{Introduction of stage-top incubator and capillary micropipette to SECM}

The beating rate of cardiomyocytes is significantly affected by environmental factors such as temperature or $\mathrm{pH}^{27,28}$ To accurately analyze the beating of cardiomyocytes by SECM, a stage-top incubator with a chamber was necessary. However, there have not been any reports on a SECM with a chamber ${ }^{15,26,33,34}$ because it is very difficult to control the microelectrode position in a sealed space. Therefore, we constructed a sealed chamber that enabled free movement of the microelectrode, it was set on a metal base to avoid prevention of stage movement (Fig. 1A). In addition, sensors for carbon dioxide $\left(\mathrm{CO}_{2}\right)$ and humidity were set in the chamber. The temperature of the culture medium and the $\mathrm{CO}_{2}$ concentration in the sealed chamber were maintained within a margin of error of $\pm 0.5^{\circ} \mathrm{C}$ and $\pm 0.3 \%$ for 4 hours, respectively (Fig. S1A). Humidity was maintained at $99.9 \%$ (data not shown). We monitored the beating motion of rat cardiomyocytes incubated in this chamber by measuring microelectrode current with a time resolution of $10 \mathrm{~ms}$ to detect beating motions in the range from 200 to 500 ms. $^{35,36}$ The spatial resolution of our SECM was previously described ${ }^{15}$ and could detect motions smaller than $1 \mu \mathrm{m}$. Because the microelectrode current value primarily depends on the distance between the microelectrode and the cell surface, ${ }^{15}$ when the microelectrode was set above a beating cardiomyocyte, the current waveform corresponding to the beating motion was continuously measured (Fig. 1B). These results demonstrated that the microelectrode position could be controlled in the sealed chamber and confirmed that the beating rate was constant for at least 2 hours (Fig. 1B).

Opening the sealed chamber causes vibrations and electrical noise that prevent the SECM from measuring cardiomyocytes beating. Thus, drug stimulation should be performed in a sealed chamber without contact. We have developed a capillary micropipette (MP) connected to SECM. ${ }^{32}$ We constructed the MP in a sealed chamber (Fig. 1A) to perform beating measurements and drug stimulation simultaneously. The MP was positioned obliquely upward of the microelectrode. The drug amount delivered from the MP was controlled by feedback control system using microelectrode current signals. To examine drug delivery performance and electrical noise in the sealed chamber, we monitored the diffusion of the delivered solution. When the delivery volume was set much larger than what was to be delivered to cells, the delivered solution spread by up to about $250 \mu \mathrm{m}$ radii (Fig. S1B). Furthermore, because no noise current was detected during drug delivery (data not shown), our SECM-MP system has the potential for the measurement of contraction kinetics during drug stimulation under cell culture conditions. 


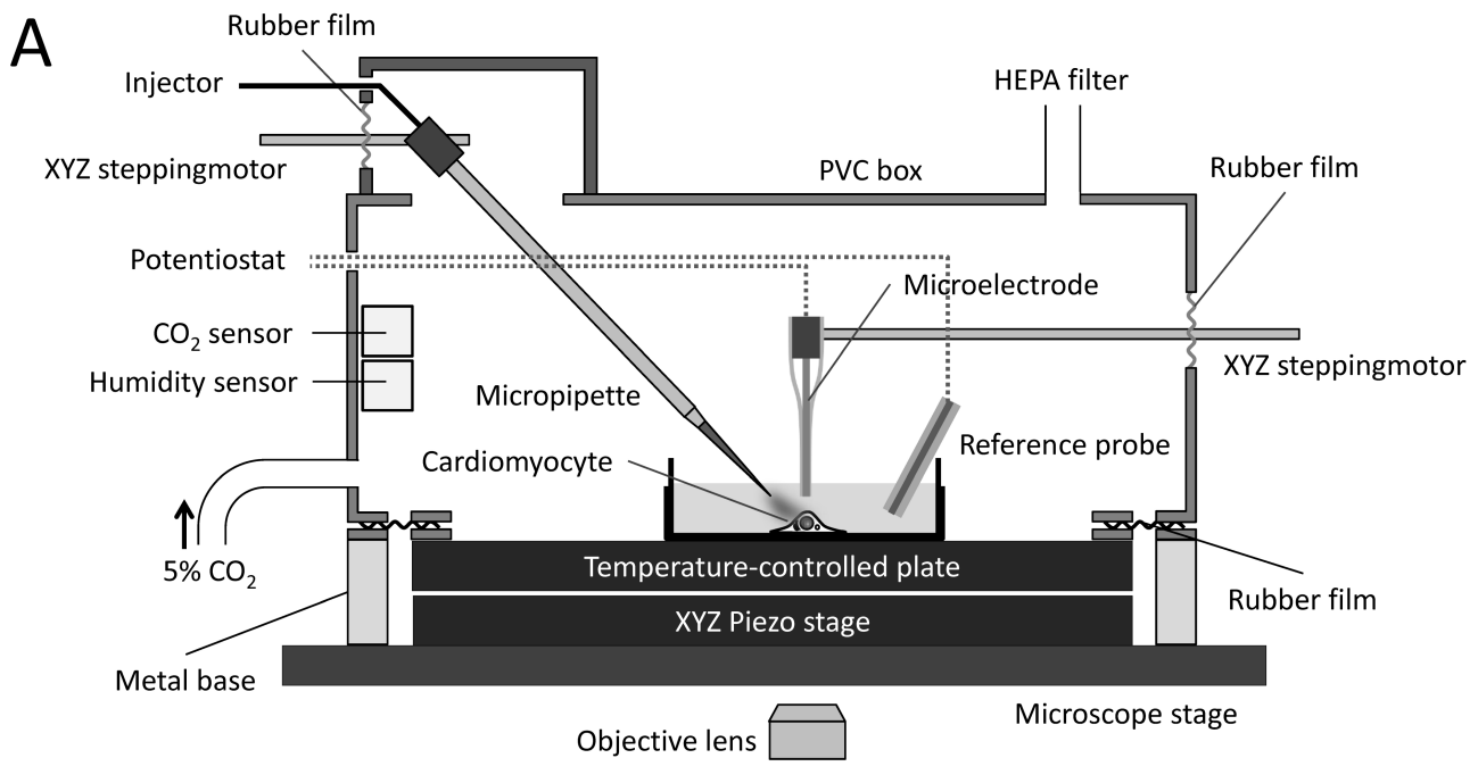

B

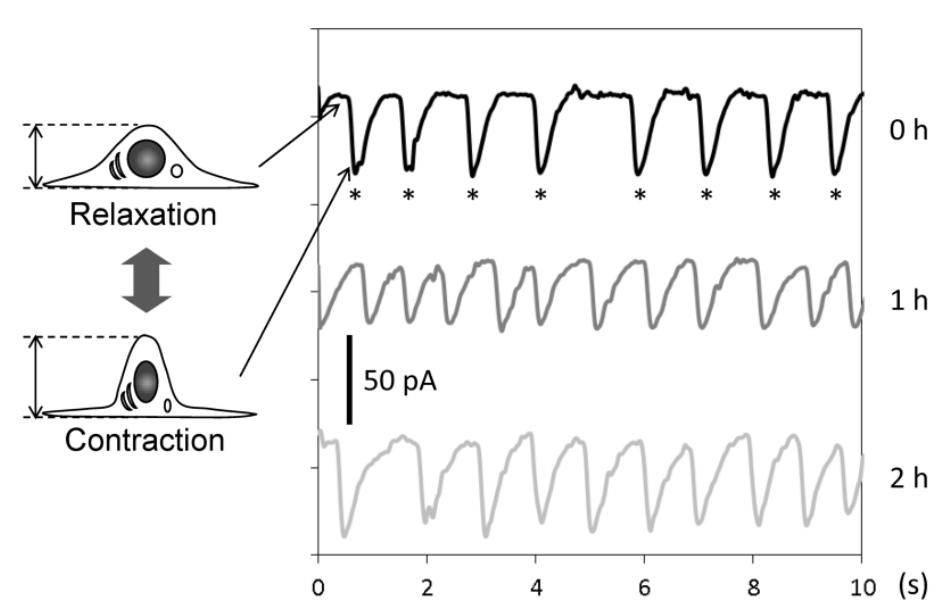

Fig. 1

(A) Schematic diagram of SECM and the MP system.

(B) Measurements of cardiac beating motion for 2 hours. The waveforms are plots of the current data corresponding to the cardiomyocytes motion. The scale bar, shown in the panel, indicates the reference value for current changes. Asterisks indicate peaks of waveforms that pinpoint the most contracted state of the cell. 


\section{Analysis of beating fluctuations of micropatterned cardiomyocytes in response to ATP delivered by the MP}

As previously reported, ${ }^{37,} 38$ since adenosine triphosphate (ATP) enhances the contractility of cardiomyocytes, beating rate was increased by ATP added to the entire medium at $50 \mu \mathrm{M}$ ("ATP” in Fig. S2A). However, when ATP was delivered from the MP, targeting a single cardiomyocyte that was cultured in the 35-mm culture dish, no beating fluctuations were induced ("ATP-MP” in Fig. S2A), even though the ATP concentration around the target cell was approximately $500 \mu \mathrm{M}$. It was unclear why locally added ATP did not affect the rhythm of the cardiomyocytes in the culture dish. When we focused on the synchronization of the beating rhythm of the cardiomyocytes in the culture dish, ${ }^{39,40}$ we hypothesized that synchronous signals from neighboring cells of the target cell might counteract the effects of the added ATP (Fig. 2A upper-panel).

To isolate a target cardiomyocyte from the synchronous rhythm in the culture dish, we introduced a micropatterning tool $^{41}$ that enabled us to make various sizes $(80-1000 \mu \mathrm{m}$ in diameter) of disc-shaped cell islands in the 35-mm culture dish (Fig. 2A lower-panel and Fig. 2B). Four days after seeding the cardiomyocytes, it was observed that cardiomyocytes in the same island were synchronized whereas each island was mutually independent. When ATP was delivered using the MP to a cellular island, the beating rate was significantly increased by approximately 2.5 times compared to the initial beating rate ("ATP-MP+island" in Fig. 2C, and Fig. S2B). The calculated ATP concentration around the island was only $20 \mu \mathrm{M}$ at most. The beating rate did not change when only buffer solution was delivered ("PBS(-)-MP+island" in Fig. 2C and Fig. S2C). These results indicated that the SECM-MP system could reproduce the same result as that of conventional addition of ATP to the entire medium (Fig. S2A). Furthermore, the beating of cardiomyocytes in an island 225-500 $\mu \mathrm{m}$ in diameter increased in response to ATP solution delivery, whereas no response was seen in $1000 \mu \mathrm{m}$ islands (data not shown). Cardiomyocytes in islands smaller than $140 \mu \mathrm{m}$ did not beat before ATP delivery. These sizes of cellular island were approximately consistent with the spread area of the locally delivered drugs (Fig. S1B). Thus, it was revealed that to obtain the effect of drug stimulation using the MP, the drug solution should spread over the target cellular island that contains one group of synchronized cardiomyocytes.

The contraction and relaxation fluctuations were analyzed by the software program that we previously developed ${ }^{15}$ with slight modification. Briefly, a plot of current was extracted for 20 seconds each for 1 minute and averaged to a single waveform, and analyzed for the duration of motion (Fig. 2D). The calculated data showed that the relaxation duration was shortened after delivery of ATP (Fig. 2D, E and F). Therefore, it was shown that our SECM-MP system could induce a beating in response to drugs and analyze the time-course beating fluctuations before and during drug stimulation.

Drugs were locally delivered to target cells, but it was unclear whether cells in separate islands 
were affected by drugs diffusing through the culture medium. ATP was added from the MP targeting a cellular island ("drug target island" in Fig. 3A). The beating motion of the cardiomyocytes was measured at a drug target island or at another island located downstream ("downstream island" in Fig. 3A). At the drug target island, the beating rate was increased, whereas no changes were detected at the downstream island (Fig. 3B and Fig. S3). The same result was obtained when the beating fluctuation was measured at a side island ("side island" in Fig. 3A and B). These results show that the area affected by the drug could be limited around the target cells to quite a small distance. The total delivered amount of ATP in each measurement was only about $1 \mathrm{nmol}$ and it was diffused and diluted to around $0.5 \mathrm{pM}$ in the culture medium. Thus, serial treatments with multiple drugs would be possible in one culture dish by replacing the drug target island, and our SECM-MP system shows promise for application to multiple drug screening. 
A

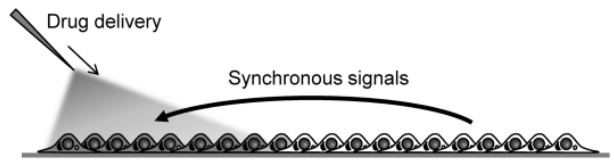

Drug delivery

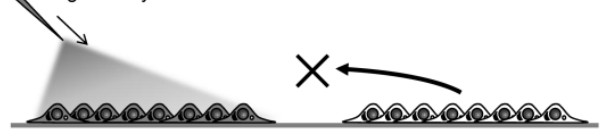

C

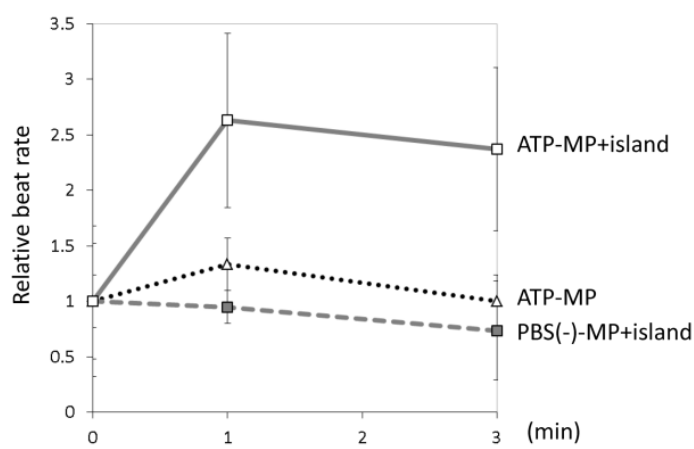

$\mathrm{E}$

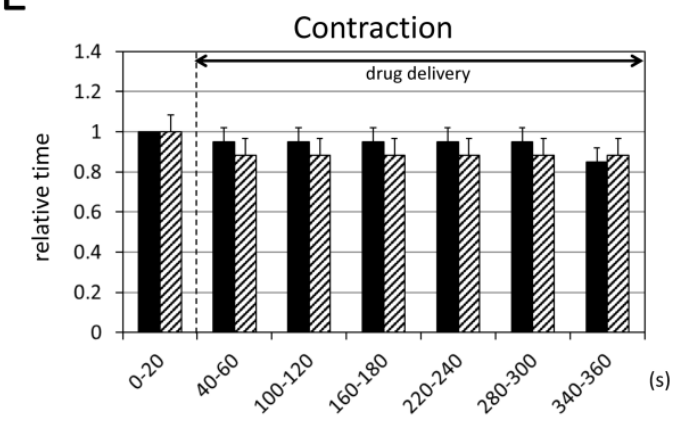

B
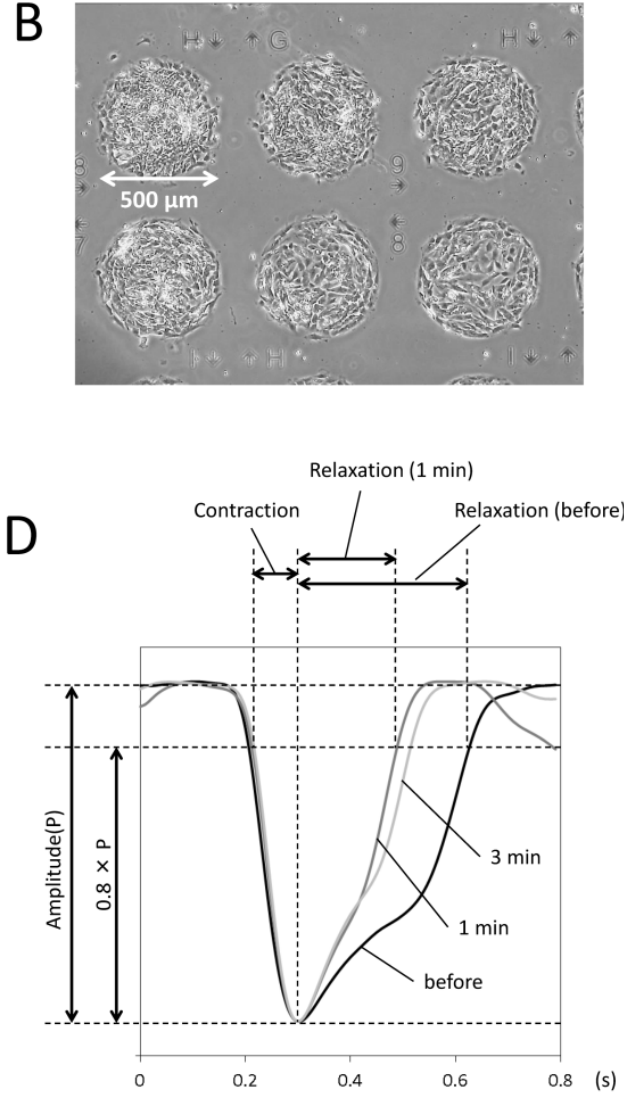

$\mathrm{F}$

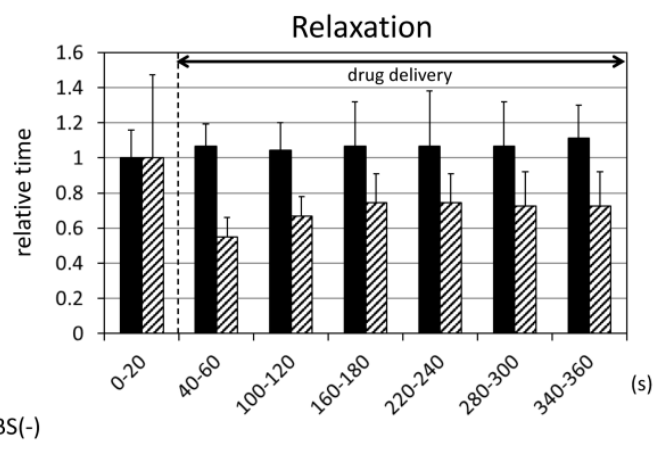

Fig. 2

Motion fluctuations of cardiomyocyte beatings induced by ATP

(A) Schematic views of desynchronization of cardiomyocytes. (B) Phase-contrast image of cardiomyocytes cultured on a micropatterned surface. The cellular islands that are $500 \mu \mathrm{m}$ in diameter are indicated. (C) Time-course of beat rates with stimulation by ATP or PBS(-) that was locally delivered. The beat rates after ATP/PBS(-) stimulation (1 and 3 min) are indicated as relative values to the beat rates before ATP/PBS(-) stimulation (0 min). (D) Comparison of the averaged 
beating waveforms before, 1 and 3 min after delivery of ATP. The duration of the contraction and relaxation were calculated based on the waveforms. (E) Contraction and (F) relaxation time of cardiomyocytes delivered with ATP or PBS(-). 0-20, 100-120, 220-240 s correspond to before (0 min), $1 \mathrm{~min}, 3 \mathrm{~min}$ respectively, as shown in (C) and (D). The relative times after ATP/PBS(-) stimulation are calculated on the basis of the time before ATP/PBS(-) stimulation. 

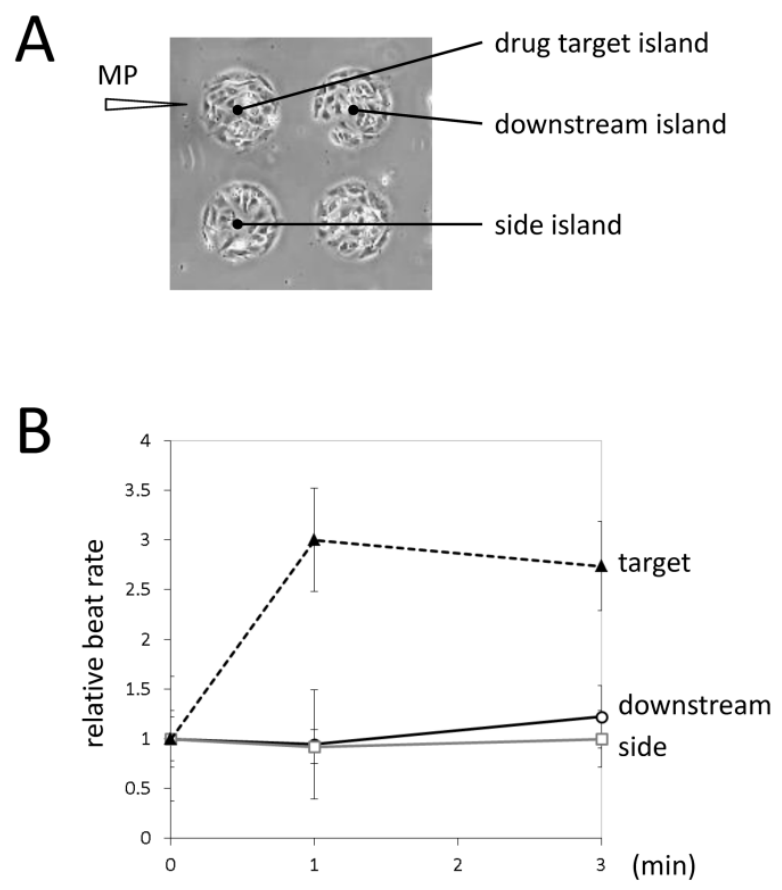

Fig. 3

Effect of ATP on neighboring islands

(A) Layout of the drug target island and the beating measurement island. Because ATP was delivered from the left, obliquely upward of the target island, the right island positioned downstream in this image. (B) Beating changes of cardiomyocytes in each island. The beat rates after ATP stimulation (1 and $3 \mathrm{~min}$ ) are indicated as relative values with the beat rates before ATP stimulation (0 min). 


\section{Application of SECM system for analyzing the effect of the cardiotoxic agent astemizole}

Astemizole has been developed as a second-generation antihistamine drug, but was withdrawn from the market due to its cardiac toxic effect. ${ }^{42}$ When we added astemizole to the entire medium using the previous SECM system, ${ }^{15}$ beating fluctuations could not be measured because the cardiomyocytes stopped beating. We then investigated whether the SECM-MP system could evaluate the toxicity of astemizole through motion fluctuations. When astemizole was added to one of the cardiomyocyte islands from the MP at $24 \mathrm{nM}$, the beating motion of the cardiomyocytes changed as the stimulation time increased (Fig. S4A). As shown in the average waveforms (Fig. 4A), calculated as for ATP previously, the duration of the contractions did not change before and after stimulation with astemizole (Fig. 4A, B), but the relaxation duration was specifically prolonged, showing a five-fold increase after 5 minutes stimulation (Fig.4A, C). No motion fluctuations were observed when delivering a control solution that did not contain astemizole (Fig. 4B, C and Fig. S4B). These motion fluctuations are consistent with the known pharmacological properties of astemizole. Astemizole is known to block cardiac potassium channels and causes prolongation of depolarization of the cardiac action potentials. ${ }^{43}$ It is called QT prolongation and may lead to sudden death. ${ }^{44}$ Because depolarization leads to contraction of the cardiomyocytes, ${ }^{45}$ QT prolongation might appear as a continuation of contraction and a delay of relaxation. Thus, we can analyze the effect of astemizole by using motion fluctuations as a replacement for the conventional electrophysiological method. Therefore, it was suggested that our SECM-MP system could evaluate the cardiotoxicity of a drug by applying beating fluctuations as an index. 


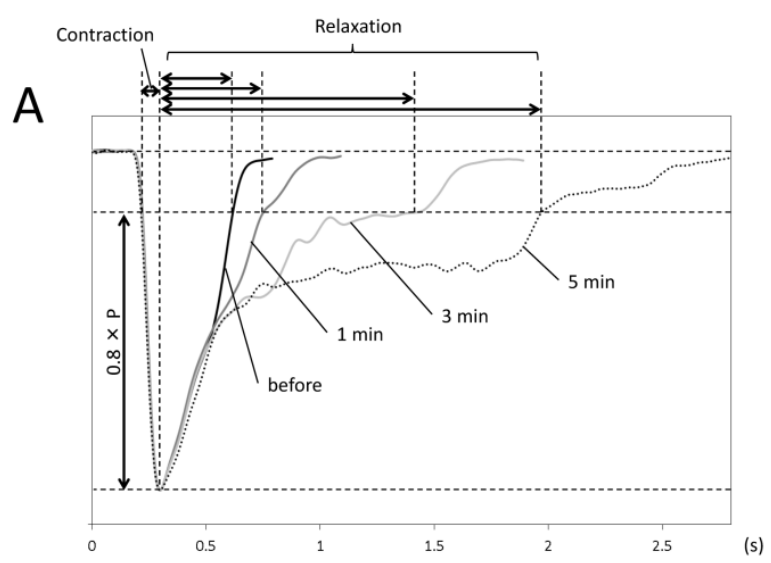

B

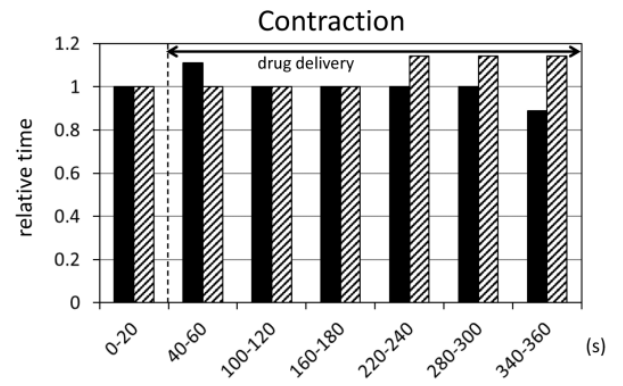

C

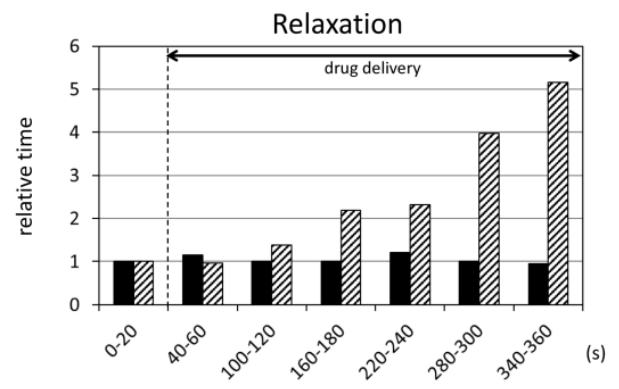

Control

$\mathbb{Z}$ Astemizole

Fig. 4

Beating changes of cardiomyocytes induced by astemizole

(A) Comparison of averaged waveforms before, $1 \mathrm{~min}, 3 \mathrm{~min}$ and $5 \mathrm{~min}$ after delivery of astemizole.

(B) Contraction and (C) relaxation times of cardiomyocytes delivered astemizole or control solutions. Control solution contains only DMSO, the solvent of astemizole. 0-20, 100-120, 220-240, 340-360 s correspond to before, $1 \mathrm{~min}, 3 \mathrm{~min}, 5 \mathrm{~min}$ respectively, as shown in (A). The relative times after astemizole/control stimulation are calculated on the basis of the time before astemizole/control stimulation. 


\section{Conclusions}

We successfully developed an analytical system for cardiac beatings and analyzed the kinetic changes of cardiomyocytes treated with the cardiotoxic agent, astemizole. We measured cardiac beating in a stable culture environment. These results proved that strict control of the microelectrode and SECM measurements could be performed even in sealed space. This suggested that our SECM system has promise in further applications of cell and biological materials. A combination of a local drug delivery system using a MP and a micropatterned surface enabled us to break the synchronous cell beating rhythm and perform beating measurements and drug stimulation simultaneously. Furthermore, because the delivered drug only affected the target cellular island, our SECM-MP system has potential for application in multiple drug screening in a single culture dish. Finally, we could analyze the kinetic changes of the cardiomyocytes upon treatment with astemizole and the result suggested a correlation between action potentials and beating motion. These results indicate that our analytical method is appropriate for cardiomyocyte analysis from a new point of view and could reveal new pharmacological properties of drugs that might be missed with conventional electrophysiological methods. 


\section{Conflicts of interest}

There are no conflicts to declare.

\section{Acknowledgments}

We thank Mr. Youhei Mukai for his supporting with the software development. We also thank Mr. Masahiro Shibuya for his helpful discussions. The financial support received, in part from the Ministry of Education, Culture, Sports, Science and Technology, Japan (Grant-in-Aid for Scientific Research (No. 17K05917)) and from the Ministry of Economy, Trade and Industry, Japan, is gratefully acknowledged. 


\section{References}

1. P. La Rochelle, J. Lexchin and D. Simonyan, Pharmaceutical Medicine, 2016, 30, 277-289.

2. I. J. Onakpoya, C. J. Heneghan and J. K. Aronson, BMC Med, 2016, 14, 10.

3. V. B. Siramshetty, J. Nickel, C. Omieczynski, B. O. Gohlke, M. N. Drwal and R. Preissner, Nucleic Acids Res, 2016, 44, D1080-1086.

4. E. G. Navarrete, P. Liang, F. Lan, V. Sanchez-Freire, C. Simmons, T. Gong, A. Sharma, P. W. Burridge, B. Patlolla, A. S. Lee, H. Wu, R. E. Beygui, S. M. Wu, R. C. Robbins, D. M. Bers and J. C. Wu, Circulation, 2013, 128, S3-13.

5. Y. Qu and H. M. Vargas, Toxicol Sci, 2015, 147, 286-295.

6. F. H. Lu, Z. Tian, W. H. Zhang, Y. J. Zhao, H. L. Li, H. Ren, H. S. Zheng, C. Liu, G. X. Hu, Y. Tian, B. F. Yang, R. Wang and C. Q. Xu, J Biomed Sci, 2010, 17, 50.

7. G. M. Dittami, S. M. Rajguru, R. A. Lasher, R. W. Hitchcock and R. D. Rabbitt, $J$ Physiol, 2011, 589, 1295-1306.

8. Z. Wang, W. Qin, Y. Shao, S. Ma, T. K. Borg and B. Z. Gao, Proc SPIE Int Soc Opt Eng, 2014, 8948.

9. J. Liu, N. Sun, M. A. Bruce, J. C. Wu and M. J. Butte, PLoS One, 2012, 7, e37559.

10. M. F. Peters, S. D. Lamore, L. Guo, C. W. Scott and K. L. Kolaja, Cardiovasc Toxicol, 2015, 15, 127-139.

11. M. M. Hossain, E. Shimizu, M. Saito, S. R. Rao, Y. Yamaguchi and E. Tamiya, Analyst, 2010, 135, 1624-1630.

12. A. Ahola, A. L. Kiviaho, K. Larsson, M. Honkanen, K. Aalto-Setala and J. Hyttinen, Biomed Eng Online, 2014, 13, 39.

13. T. Hayakawa, T. Kunihiro, T. Ando, S. Kobayashi, E. Matsui, H. Yada, Y. Kanda, J. Kurokawa and T. Furukawa, J Mol Cell Cardiol, 2014, 77, 178-191.

14. M. Maddah, J. D. Heidmann, M. A. Mandegar, C. D. Walker, S. Bolouki, B. R. Conklin and K. E. Loewke, Stem Cell Reports, 2015, 4, 621-631.

15. Y. Hirano, M. Kodama, M. Shibuya, Y. Maki and Y. Komatsu, Anal Biochem, 2014, 447, 39-42.

16. C. Zhao, J. K. Sinha, C. A. Wijayawardhana and G. Wittstock, Journal of Electroanalytical Chemistry, 2004, 561, 83-91.

17. Y. Hirano, T. Yasukawa, Y. Mase, D. Oyamatsu, H. Shiku, F. Mizutani and T. Matsue, Electrochemistry, 2012, 80, 30-32.

18. B. Liu, S. A. Rotenberg and M. V. Mirkin, Proc Natl Acad Sci U S A, 2000, 97, 9855-9860.

19. M. M. Zhang, Y. T. Long and Z. Ding, J Inorg Biochem, 2012, 108, 115-122. 
20. M. Nebel, S. Grutzke, N. Diab, A. Schulte and W. Schuhmann, Faraday Discussions, 2013, 164, 19-32.

21. D. Koley and A. J. Bard, Proc Natl Acad Sci U S A, 2010, 107, 16783-16787.

22. Y. Matsumae, T. Arai, Y. Takahashi, K. Ino, H. Shiku and T. Matsue, Chem Commun (Camb), 2013, 49, 6498-6500.

23. Y. Hirano, Y. Nishimiya, K. Kowata, F. Mizutani, S. Tsuda and Y. Komatsu, Analytical Chemistry, 2008, 80, 9349-9354.

24. M. M. Zhang, Y. T. Long and Z. Ding, Chem Cent J, 2012, 6, 20.

25. Y. Takahashi, A. I. Shevchuk, P. Novak, B. Babakinejad, J. Macpherson, P. R. Unwin, H. Shiku, J. Gorelik, D. Klenerman, Y. E. Korchev and T. Matsue, Proc Natl Acad Sci $U S A, 2012,109,11540-11545$.

26. J. G. Ummadi, V. S. Joshi, P. R. Gupta, A. K. Indra and D. Koley, Anal Methods, 2015, 7, 8826-8831.

27. J. van Der Velden, L. J. Klein, R. Zaremba, N. M. Boontje, M. A. Huybregts, W. Stooker, L. Eijsman, J. W. de Jong, C. A. Visser, F. C. Visser and G. J. Stienen, Circulation, 2001, 104, 1140-1146.

28. Y. Fu, G. Q. Zhang, X. M. Hao, C. H. Wu, Z. Chai and S. Q. Wang, Biophys J, 2005, 89, 2533-2541.

29. H. Liu, W. Qin, Y. Shao, Z. Ma, T. Ye, T. Borg and B. Z. Gao, J Biomed Opt, 2011, 16, 126012 .

30. H. Liu, Y. Shao, W. Qin, R. B. Runyan, M. Xu, Z. Ma, T. K. Borg, R. Markwald and B. Z. Gao, Cardiovasc Res, 2013, 97, 262-270.

31. V. De Arcangelis, S. Liu, D. Zhang, D. Soto and Y. K. Xiang, Mol Pharmacol, 2010, 78, 340-349.

32. Y. Hirano, K. Kowata, M. Kodama and Y. Komatsu, Bioelectrochemistry, 2013, 92, $1-5$.

33. D. Koley and A. J. Bard, Proc Natl Acad Sci US A, 2012, 109, 11522-11527.

34. S. E. Salamifar and R. Y. Lai, Anal Chem, 2013, 85, 9417-9421.

35. S. Nattel and L. Carlsson, Nature Reviews Drug Discovery, 2006, 5, 1034-1049.

36. S. Tsukamoto, T. Fujii, K. Oyama, S. A. Shintani, T. Shimozawa, F. Kobirumaki-Shimozawa, S. Ishiwata and N. Fukuda, Journal of General Physiology, 2016, 148, 341-355.

37. R. S. Danziger, S. Raffaeli, R. Moreno-Sanchez, M. Sakai, M. C. Capogrossi, H. A. Spurgeon, R. G. Hansford and E. G. Lakatta, Cell Calcium, 1988, 9, 193-199.

38. F. Scamps and G. Vassort, Pflugers Arch, 1990, 417, 309-316.

39. I. Harary and B. Farley, Exp Cell Res, 1963, 29, 451-465. 
40. G. E. Mark and F. F. Strasser, Experimental Cell Research, 1966, 44, 217-233.

41. A. Deglincerti, F. Etoc, M. C. Guerra, I. Martyn, J. Metzger, A. Ruzo, M. Simunovic, A. Yoney, A. H. Brivanlou, E. Siggia and A. Warmflash, Nat Protoc, 2016, 11, 2223-2232.

42. Y. G. Yap and A. J. Camm, Heart, 2003, 89, 1363-1372.

43. Z. Zhou, V. R. Vorperian, Q. Gong, S. Zhang and C. T. January, J Cardiovasc Electrophysiol, 1999, 10, 836-843.

44. S. Nachimuthu, M. D. Assar and J. M. Schussler, Ther Adv Drug Saf, 2012, 3, 241-253.

45. I. Y. Kuo and B. E. Ehrlich, Cold Spring Harb Perspect Biol, 2015, 7, a006023. 